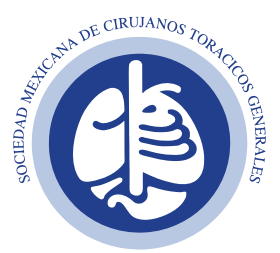

Vol. 1, Núm. 2

Mayo-Agosto 2020

pp 39-40

REVISTA

MEXICANA DE

CIRUGÍA

TORÁCICA

GENERAL

doi: $10.35366 / 101148$

\author{
Editorial
}

\title{
El cirujano de tórax y el SARS-CoV-2
}

\author{
Thoracic surgeon and SARS-CoV-2
}

Graciano Castillo-Ortega, * Ulises Loyola-García, ${ }^{\ddagger}$ Miguel Alejandro Martínez-Arias, ${ }^{\$}$

Gustavo Félix Salazar-Otaola," Carlos A Olivares-Torres"

La pandemia del SARS-CoV-2 ha tenido un impacto sin precedentes a nivel mundial; el Área de la Cirugía de Tórax no ha sido la excepción, a partir de marzo del 2020 en nuestro país, con la reconversión hospitalaria, el cirujano de tórax se ha vuelto protagonista en esta nueva enfermedad.

Los Servicios de Cirugía de Tórax y de Cirugía General a lo largo del país, al igual que las guías y algoritmos, han ido cambiando a través de la enfermedad para la seguridad del paciente y de los propios cirujanos de tórax, quienes en lugar de realizar cirugía de invasión mínima para los diferentes padecimientos, tuvieron que adaptarse a las condiciones actuales participando de manera activa en la resolución de complicaciones por COVID-19; así pues, se convirtieron en cirujanos de traqueostomías a pesar del riesgo de contagio que implica para todo el personal, ya que la cirugía habitual disminuyó considerablemente, y en algunos hospitales prácticamente desapareció.

A través de la misma pandemia se ha participado de manera activa en la resolución de las complicaciones de esta enfermedad, y vemos cómo los pacientes que se trataban de otras patologías antes de la pandemia han desaparecido por el confinamiento y por el temor de ir a los hospitales COVID.

En el camino se han infectado varios cirujanos y otros han sucumbido a la enfermedad,

* Cirujano de tórax adscrito al Servicio de Cirugía, Hospital General del Estado de Sonora, Hermosillo.

${ }^{\ddagger}$ Cirujano de tórax.

Subdirector del Centro Médico ISSEMyM, Toluca.

$\S$ Cirujano de tórax.

Jefatura de Cirugía de

Tórax y Neumología.

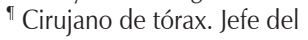

Servicio de Cirugía, Hospital General de Tijuana.

II Cirujano de tórax. Jefe del Servicio de Cirugía de tórax. Hospital General de Tijuana.

Correspondencia:

Dr. Carlos A Olivares-Torres E-mail: editor.revista@smctg.org pero eso no nos ha detenido para continuar manejando a todos los pacientes y poco a poco al estar en la "llamada nueva normalidad". Con mucho esfuerzo, vocación, voluntad y cuidados hemos tenido que operar y reabrir los Servicios de Cirugía de Tórax del país, para poder darle atención a todos los pacientes que por el confinamiento o infección no pudieron ser atendidos ya con personal médico vacunado, pacientes con mejores protocolos de seguridad para sus procedimientos y con mejor conocimiento de la enfermedad. Sin embargo, el advenimiento de escaladas de infección en la población provocará el cierre en instituciones que sólo se dedicarán a atender al SARS-CoV-2.

Seguramente esta pandemia nos enseñará a tratar con más cuidado a todos los pacientes de Patología Torácica con o sin relación a enfermedad por COVID-19; así como a no olvidar los principios generales de higiene, técnica estéril y vestido quirúrgico adecuado

Citar como: Castillo-Ortega G, Loyola-García U, Martínez-Arias MA, Salazar-Otaola GF, Olivares-Torres CA. El cirujano de tórax y el SARS-CoV-2. Rev Mex Cir Torac Gen. 2020: 1(2); 39-40. https://dx.doi.org/10.35366/101148 
para evitar contaminaciones durante esta enfermedad que parece no tener fin, y que viene en oleadas a las cuales hay que enfrentar con la resiliencia y conocimiento que todo cirujano de tórax tiene dentro de sí.

Regresaremos un día a la normalidad con el SARSCoV-2 endémico y con la comprensión de cómo cuidarnos de la enfermedad con las recomendaciones y el liderazgo que se tiene y se tendrá a lo largo de esta pandemia, y el cirujano de tórax estará a la vanguardia del tratamiento de la patología torácica y las secuelas que nos dejará la COVID-19, ya sea con la realización de una traqueostomía o siendo la única opción de vida un trasplante pulmonar.

Esta pandemia ha marcado un antes y un después en nuestras vidas y en el quehacer y la práctica de la cirugía torácica general, no sólo en México, sino en el mundo entero. El cirujano se ha convertido en parte fundamental del tratamiento de SARS-CoV-2. 